\title{
SS433's Jet Trace from ALMA Imaging and Global Jet Watch Spectroscopy: Evidence for Post-launch Particle Acceleration
}

\author{
Katherine M. Blundell ${ }^{1}$ (1D, Robert Laing ${ }^{2}$, Steven Lee ${ }^{3}$, and Anita Richards ${ }^{4}$ \\ ${ }^{1}$ University of Oxford, Department of Physics, Keble Road, Oxford OX1 3RH, UK \\ ${ }^{2}$ SKA Organisation, Jodrell Bank Observatory, Lower Withington, Macclesfield SK11 9DL, UK \\ ${ }^{3}$ Anglo-Australian Telescope, Coonabarabran NSW 2357, Australia \\ ${ }^{4}$ Jodrell Bank Centre for Astrophysics, School of Physics and Astronomy, University of Manchester, Manchester M13 9PL, UK \\ Received 2018 October 2; accepted 2018 October 15; published 2018 November 5
}

\begin{abstract}
We present a comparison of Doppler-shifted $\mathrm{H} \alpha$ line emission observed by the Global Jet Watch from freshly launched jet ejecta at the nucleus of the Galactic microquasar SS433 with subsequent Atacama Large Millimeter/ submillimeter Array (ALMA) imaging at mm-wavelengths of the same jet ejecta. There is a remarkable similarity between the transversely resolved synchrotron emission and the prediction of the jet trace from optical spectroscopy: this is an a priori prediction and not an a posteriori fit, confirming the ballistic nature of the jet propagation. The mm-wavelength of the ALMA polarimetry is sufficiently short that the Faraday rotation is negligible and therefore that the observed $\boldsymbol{E}$-vector directions are accurately orthogonal to the projected local magnetic field. Close to the nucleus, the $\boldsymbol{B}$-field vectors are perpendicular to the direction of propagation. Further out from the nucleus, the $\boldsymbol{B}$-field vectors that are coincident with the jet instead become parallel to the ridge line; this occurs at a distance where the jet bolides are expected to expand into one another. X-ray variability has also been observed at this location; this has a natural explanation if shocks from the expanding and colliding bolides cause particle acceleration. In regions distinctly separate from the jet ridge line, the fractional polarization approaches the theoretical maximum for synchrotron emission.
\end{abstract}

Key words: acceleration of particles - binaries: close - stars: individual: (SS433) - stars: jets

\section{Introduction}

Since shortly after its discovery four decades ago, the prototypical Galactic microquasar SS433 has been known to eject oppositely directed jets whose launch axis precesses with a cone angle of about $19^{\circ}$ approximately every 162 days, and whose speeds average to about a quarter of the speed of light. Striking images of the emission at radio $(\mathrm{cm})$ wavelengths reveal a zigzag/corkscrew structure that is generated because of the above properties modulated by light-travel time effects arising from its orientation with respect to our line of sight (Hjellming \& Johnston 1981; Stirling et al. 2002; Blundell \& Bowler 2004; Miller-Jones et al. 2008; Roberts et al. 2008). The optical spectra of this object are characterized by a strong Balmer $\mathrm{H} \alpha$ emission line complex close to the rest-wavelength of this line, and also blueshifted and redshifted lines whose observed wavelengths change successively on a daily basis according to the instantaneous speed and angle of travel with respect to our line of sight. Fitted parameters to the kinematic model developed from the first few years of optical spectroscopy were presented by, for example, Margon (1984) and Eikenberry et al. (2001). Previously the timing of optical spectroscopy and spatially resolved radio imaging has not permitted the observation of the same ejecta both at launch and after propagation. We present the first mm-wave image of SS433 from the Atacama Large Millimeter/submillimeter Array (ALMA) in combination with optical spectroscopy (Section 2) of the same ejecta observed during the year prior to the ALMA observations (Section 3). This allows us to distinguish ballistic motion post-launch from deceleration (e.g., Stirling et al. 2004).

A longstanding question is why SS433's jet ejecta are primarily line emitting at launch yet synchrotron emitting at larger distances from the nucleus; the polarization changes explored in Section 5 shed light on this. Inference of the magnetic-field structure in the jets is complicated by the combined effects of Faraday rotation and time-variable structure. Previous studies (Stirling et al. 2004; Miller-Jones et al. 2008; Roberts et al. 2008) have been hampered by lack of resolution and frequency coverage as well as the uncertain effects of spatial and temporal variations in Faraday rotation. The dependence of Faraday rotation on the square of the wavelength $(\lambda)$ means that wide-band observations at $\mathrm{mm}$ wavelengths allow the projected field direction to be determined accurately in a single observation even close to the core, where Faraday rotation measures may be large (Roberts et al. 2008). We present our polarimetric $230 \mathrm{GHz}$ results in Section 5 .

\section{Optical Spectroscopy and Inference from Doppler Shifts}

Spectra of SS433 spanning a wavelength range of approximately 5800 to 8500 Angstroms and with a spectral resolution of 4000 were observed in the year prior to the ALMA observations whenever this target was a nighttime object. These were carried out with the multi-longitude Global Jet Watch telescopes, each of which is equipped with an Aquila spectrograph; the design and testing of these high-throughput spectrographs are described by S. Lee et al. (2018, in preparation). The observatories, astronomical operations, processing, and calibration of the spectroscopic data streams are described in K. M. Blundell et al. (2018, in preparation). Almost all of these spectra contain a pair of so-called "moving lines" arising from the most recently launched jet bolides in SS433. The wavelengths corresponding to the centroids of the blueshifted and redshifted $\mathrm{H} \alpha$ emission were converted into 
redshift pairs with respect to $\mathrm{H} \alpha$ in the rest frame of SS433 according to its systemic velocity with respect to Earth (Lockman et al. 2007). The launch speed of each pair of bolides was derived from these redshift and blueshift pairs for a given spectrum (Blundell \& Bowler 2005, Equation (2)). This avoids the approximation of constant ejection speed, which has been shown to be inaccurate from archival spectroscopy (Blundell \& Bowler 2005; Blundell et al. 2011). Assuming that the subsequent motion is ballistic (this assumption is discussed in Section 4), and adopting the standard kinematic model (Hjellming \& Johnston 1981), the locations that they attain by the Julian Date of the midpoint of the ALMA observations (2457294.4836) are calculated and plotted in Figure 1. The assumed parameters of the kinematic model using the notation of Eikenberry et al. (2001) and Hjellming \& Johnston (1981) are: cone angle $\theta=19^{\circ}$ (Hjellming \& Johnston use $\psi$ ), inclination $i=79^{\circ}$, rotation on the sky $\chi=10^{\circ}$ (position angle $+100^{\circ}$ ), period $P=162.34$ day (K. M. Blundell et al. 2018, in preparation) and distance $d=5.5 \mathrm{kpc}$ (Blundell \& Bowler 2004; Lockman et al. 2007). The ejection phase was determined by fitting to the observed redshift pairs from JD 2457000 to JD 2457293.5. The phase $\phi=(2 \pi / P)\left(t-t_{\text {ref }}\right)+\phi_{0}$ with $\phi_{0}=-0.241$ rad for a reference Julian date of $t_{\text {ref }}=2456000$. $\phi$ is used as in Equation (1) of Eikenberry et al. (2001), and Hjellming \& Johnston (1981) denote the same quantity by $\Omega\left(t_{0}-t_{\text {ref }}\right)$.

\section{Millimeter Polarimetric Imaging}

SS433 was observed using 27 ALMA antennas between 2015 September 28 21:26 and September 29 01:46 UT. Three execution blocks were run almost in sequence and under similar conditions. The precipitable water vapor column was around $1.4 \mathrm{~mm}$. The correlator was set up in Time Division Multiplex mode with a total bandwidth of $7.5 \mathrm{GHz}$, in four $1.75 \mathrm{GHz}$ spectral windows (SPWs) centered at 224, 226, 240, and $242 \mathrm{GHz}$. Each SPW was divided into 64 spectral channels and $\mathrm{XX}, \mathrm{YY}, \mathrm{XY}$, and YX correlations were recorded. The longest and shortest baselines were 2270 and $43 \mathrm{~m}$, sensitive to angular scales $\lesssim 3.7$ arcsec.

The quasar J1751+0939 was used as a bandpass, polarization, and flux scale calibrator and $\mathrm{J} 1832+0731$ was used as the phase-reference source on an approximately 8 minutes cycle. The total integration time on SS433 was $\approx 2 \mathrm{hr}$. Initial data reduction followed standard ALMA scripts, executed in CASA (Schnee et al. 2014). The flux density of J1751+0939 during these observations was taken to be $3.7275 \mathrm{Jy}$ at $232.86 \mathrm{GHz}$ with a spectral index $\alpha=-0.441$ (defined in the sense $\left.S(\nu) \propto \nu^{-\alpha}\right)$ and the total flux scale uncertainty is about $10 \%$. Polarization leakage was calibrated as described by Nagai et al. (2016). Several iterations of CLEAN in multi-frequency synthesis mode (Rau \& Cornwell 2011) followed by selfcalibration were used to improve the imaging of SS433. The final iteration of amplitude and phase self-calibration was made by combining all four spectral windows using a model with two Taylor series terms. We show the zero-order Taylor series $I$ image after self-calibration, together with polarized intensity images derived from $Q$ and $U$ for the entire band (we demonstrate below that Faraday rotation is negligible for our frequency range). The off-source rms levels are 13,11, and $12 \mu \mathrm{Jy}$ beam $^{-1}$ in $I, Q$, and $U$, respectively, consistent with the expectations for thermal noise alone. The restoring beam has FWHM $0.19 \times 0.16 \operatorname{arcsec}^{2}$.
The I image (Figure 1, central panel, grayscale) shows the familiar zigzag/corkscrew shape of SS433. The peak flux density at $233 \mathrm{GHz}$ is $86.0 \mathrm{mJy}^{\text {beam }}{ }^{-1}$. The in-band spectral index of the core is $-0.29 \pm 0.14$.

\section{Comparison of Time-extrapolated Spectroscopy with ALMA Imaging}

Figure 1 shows excellent agreement between the jet trace predicted by the redshift and blueshift pairs from the optical spectroscopy and the brightness distribution subsequently measured by ALMA at mm-wavelengths. There are only three free parameters in the superposition: two positional offsets to align the ejection center with the peak of the radio emission, and the rotation of the precession axis on the sky (which was taken from Hjellming \& Johnston 1981 and not determined independently). This is the first time that it has been possible to demonstrate the superposition directly with optical spectroscopy covering the appropriate time period. We find no evidence for significant deceleration of the jet post-launch (which would be evinced by radio emission systematically lagging the optical bolides). In particular, we can rule out a deceleration of $0.02 c$ just outside of the launch location as suggested ${ }^{5}$ by Stirling et al. (2004). This would lead to a systematic offset of $\approx 0.3 \operatorname{arcsec}$ between the predicted jet trace and the ALMA brightness distribution at projected distances $\gtrsim 1$ arcsec, most obviously on the East side of the source; this is not seen. More stringent constraints on deceleration can be obtained from a comparison of the jet trace predicted by optical spectroscopy with Very Large Array (VLA) radio imaging between 8 and $12 \mathrm{GHz}$, in which the trace is detectable out to much greater distances from the nucleus than is possible in our current $230 \mathrm{GHz}$ observations. We will address this comparison in a future paper, together with possible correlations of speed with launch angle.

\section{5. $B$-field Structure}

The apparently conflicting results in the literature for the relation between the projected magnetic-field direction and the underlying jet flow in SS433 can be understood by consideration of the different distances from the nucleus probed by these studies. Stirling et al. (2004) and Roberts et al. (2008) found a preferential alignment between the magnetic field and the jet ridge line from $\approx 0.4-2$ arcsec from the nucleus, whereas Figure 8 of Miller-Jones et al. (2008) suggests that the field is instead parallel to the ballistic velocity of the jet knots at distances larger than 2 arcsec.

Our measurements of the magnetic-field direction are much less affected by Faraday rotation than those in earlier work. Stirling et al. (2004) found a mean rotation measure (RM) of $119 \mathrm{rad} \mathrm{m}^{-2}$ (excluding the core), which implies a position angle rotation of $0^{\circ} .01$ at $230 \mathrm{GHz}$. Even for the RMs of $\approx 600 \mathrm{rad} \mathrm{m}^{-2}$ estimated for distances within 0.4 arcsec of the core (Stirling et al. 2004; Roberts et al. 2008), the inferred rotation is still only $0^{\circ} .06$ at this ALMA band. We also find no evidence for any wavelength-dependent rotation across our observing band. In particular, the position angles measured for the individual SPWs at the location of the core (where we might expect maximum Faraday rotation) are consistent with the mean value for the band, with an rms scatter of 1.0 ;

\footnotetext{
5 The lower speeds suggested by Stirling et al. (2004) are a direct consequence of their adoption of a smaller distance $d=4.8 \mathrm{kpc}$ for SS433.
} 

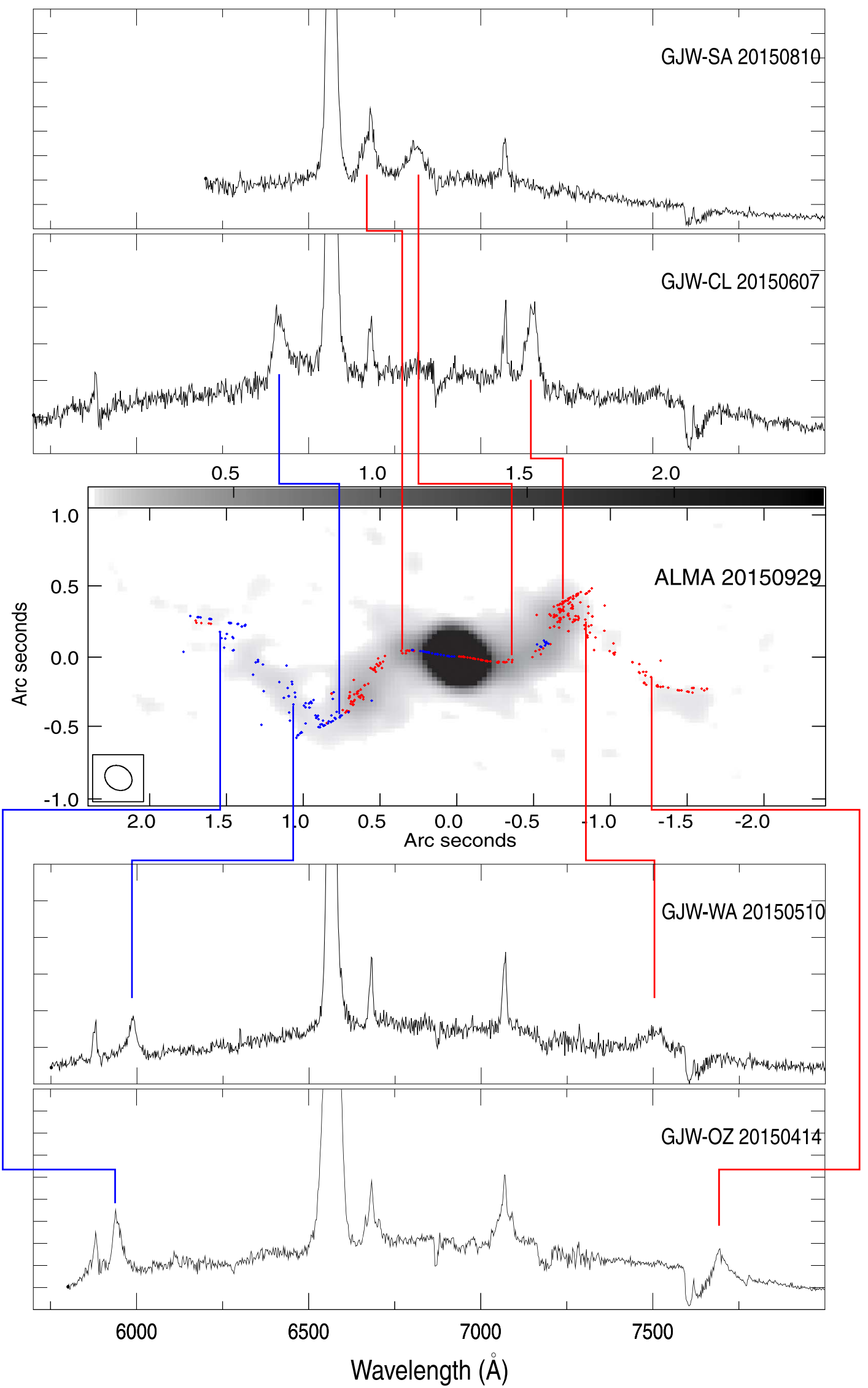

Figure 1. Central panel: mm-wave image made from ALMA observations in 2015 September described in Section 3 overlaid with symbols representing the positions attained by the bolides whose speeds are measured via optical spectroscopy and assumed to move ballistically after launch. Upper two and lower two panels: representative spectra from four different dates prior to the ALMA observations are shown, revealing a pair of Doppler-shifted lines corresponding to emission from the oppositely moving jet bolides, recently launched and still optically radiant. These spectra are from each of four different observatories; from bottom to top: eastern Australia (GJW-OZ), Western Australia (GJW-WA), Chile (GJW-CL), and South Africa (GJW-SA). 


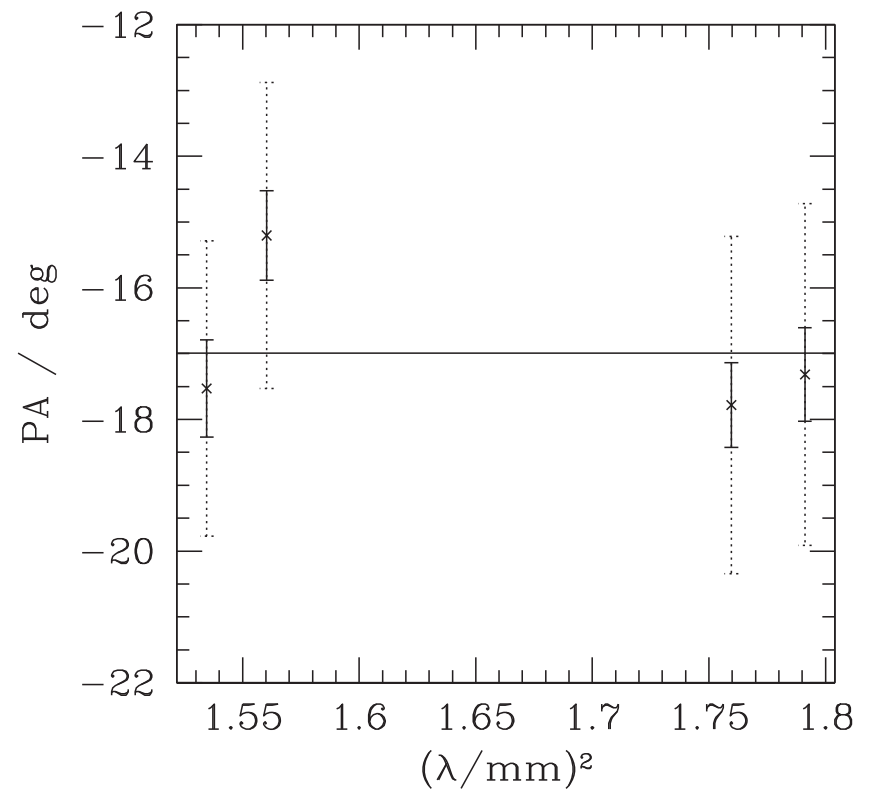

Figure 2. Direction of the $\boldsymbol{B}$-field at four different wavelengths plotted as a function of $\lambda^{2}$. All measurements are consistent with $-17^{\circ}$. The solid bars represent errors due to thermal noise alone, while the dotted bars include systematic errors.

Figure 2 shows no systematic trend. We therefore conclude that the position angles plotted in Figure 3 are not significantly affected by Faraday rotation.

While the fractional polarization of the nucleus of SS433 at $230 \mathrm{GHz}$ is low $(p=0.011)$, the position angle is still securely determined. The inset to Figure 3 shows that within $\approx 0.35$ arcsec of the nucleus the orientation of the $B$-field vectors is consistent with being perpendicular to the jet ridge line (and also to the line of radial ejection, which is indistinguishable from it at this distance), consistent with the tentative suggestion by Roberts et al. (2008). At a distance of $\approx 0.35$ arcsec, the degree of polarization increases to $p \approx 0.1$. Here, the field directions in both jets become parallel to the ridge line and clearly inconsistent with the direction of ballistic motion. This relative orientation persists out to at least 0.7 arcsec, beyond which we cannot measure accurate position angles. Our result is consistent with that of Stirling et al. (2004), but with higher angular resolution and lower uncertainties from Faraday rotation. For distances from the launch point exceeding $\approx 2$ arcsec, Miller-Jones et al. (2008) reported the magnetic field of the jet as being parallel to the local velocity vector (using the value for rotation measure reported by Stirling et al. 2004).

The transition in field direction at $0.35 \operatorname{arcsec}\left(3 \times 10^{14} \mathrm{~m}\right)$ from launch may bear on the oft-debated question of whether the outflow in SS433 is best described as a succession of independent bolides or a continuous jet. It is interesting to compare this distance with the point at which the expanding bow shocks surrounding neighboring bolides first intersect. If we assume that one bolide is ejected per day at a speed of $0.26 c$, their radial separation is $\approx 6.7 \times 10^{12} \mathrm{~m}$. If the shock expansion speed is comparable with the expansion rate of the radio knots measured with very long baseline interferometry (VLBI; $\approx 0.015 c$; Jeffrey et al. 2016), then the shock fronts will indeed expand into each other and interact when the bolides have traveled $\approx 3 \times 10^{14} \mathrm{~m}$ from the nucleus, roughly where the change in field direction occurs. Bolides will coalesce to
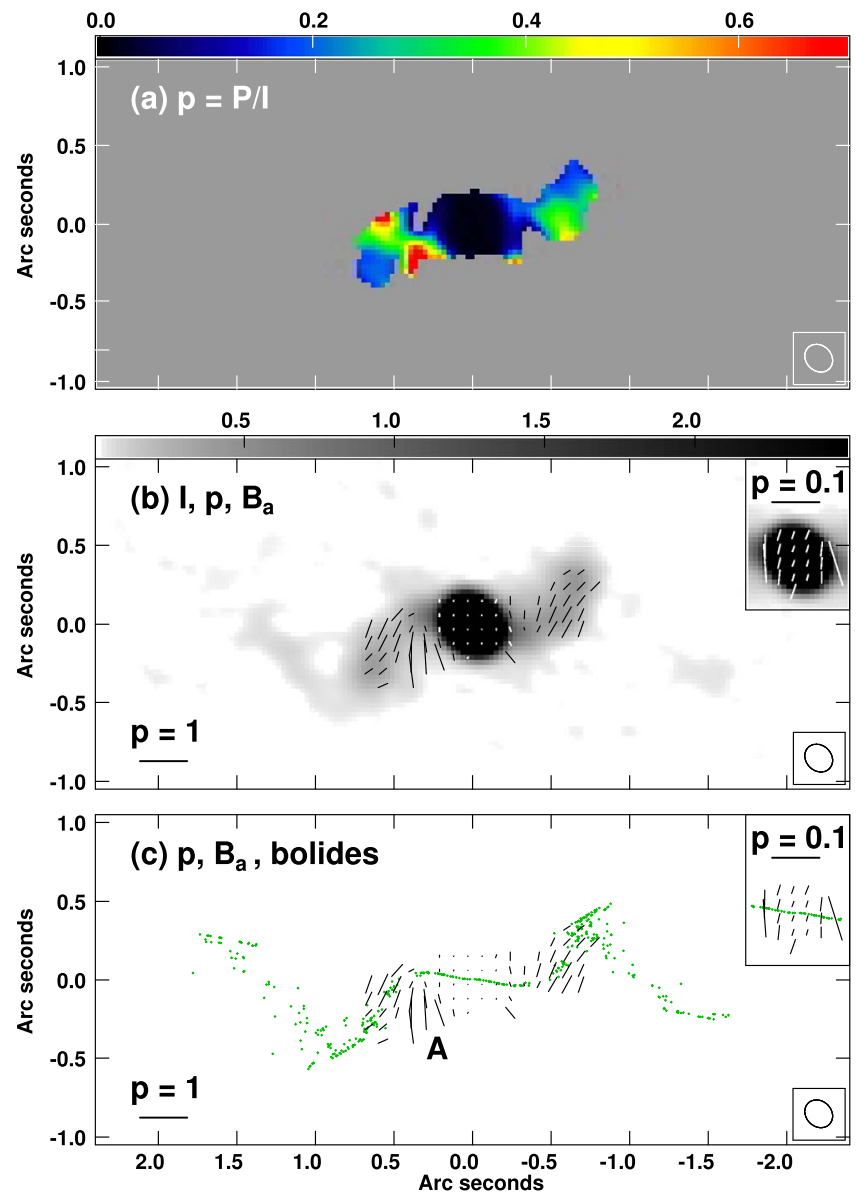

Figure 3. Panel (a) shows a color scale depicting the fractional polarization, $p$, of SS433 averaged over the ALMA observing band. Points are shown blanked (gray) wherever the total intensity $I<5 \sigma_{I}$. Panel (b) shows vectors whose lengths are proportional to $p$ (with the scale indicated by the labeled bar) and whose directions are along the apparent $\boldsymbol{B}$-field direction (i.e., rotated by $90^{\circ}$ from the $\boldsymbol{E}$-vector direction with no correction for Faraday rotation: see the text). The vectors are plotted where $I>5 \sigma_{I}$ and $P>3 \sigma_{I}$ and are superimposed on a grayscale of total intensity, as indicated by the wedge labeled in mJy beam ${ }^{-1}$. The inset shows the core with vectors plotted on an expanded scale for $I>1 \mathrm{mJy}^{-1}$ beam $^{-1}$ and $P>3 \sigma_{I}$. Panel (c): the same as panel (b), but with vectors superimposed on the inferred locations reached by pairs of plasma bolides (green crosses, as in Figure 1). The inset again shows the core region.

form larger structures which will then cease to interact with one another, when the paths of successive bolide conglomerates are too angularly divergent. Thereafter, the magnetic field observed to be associated with the jet trace will no longer reflect the details of the bolides as launched but rather their interactions with the (magnetized) medium through which they flow. This magnetic field behavior appears to dominate for distances from the launch point exceeding $\approx 2$ arcsec.

Very highly polarized emission is observed away from the jet trace on the East side of the source (labeled A in Figure 3). Both Roberts et al. (2008) and Miller-Jones et al. (2008) have drawn attention to these off-ridge line regions being significantly more polarized than the jet ridge line itself in VLA data at $15 \mathrm{GHz}$ and $8 \mathrm{GHz}$, respectively. Our $230 \mathrm{GHz}$ data show significantly higher fractional polarization values $(0.6-0.7)$ in these regions. Comparison of all these data suggest that the degree and direction of polarization may change with precession period and possibly also distance from the nucleus.

The interaction of bolides into larger coalescences has been reasoned above to occur where there is a change in polarization 
behavior, namely at approximately 0.35 arcsec from the nucleus. We note that this coincides with the region reported by Migliari et al. $(2002,2005)$ to show distinct X-ray variability that we suggest arises from shocks formed by the coalescence. Such a mechanism would naturally give rise to the stochastic nature of the X-ray variations reported by Migliari et al. $(2002,2005)$.

\section{Conclusions}

More than one precession period of the jets in the Galactic microquasar SS433 is shown to be traced out at mmwavelengths in our ALMA imaging. This shows remarkable concordance with the predicted trace of the same ejecta from Global Jet Watch spectroscopy at earlier epochs.

At mm-wavelengths the Faraday rotation toward SS433 is negligible. By 0.35 arcsec from the nucleus the $\boldsymbol{B}$-field direction has changed from being perpendicular to parallel to the jet ridge line. This occurs where bolides are expected to have expanded into one another, and where X-ray variability has been reported, consistent with the onset of particle acceleration and the change from line emission at launch to dominant synchrotron emission further out.

This Letter makes use of the following ALMA data: ADS/ JAO.ALMA\#2013.1.01369.S. ALMA is a partnership of ESO (representing its member states), NSF (USA) and NINS (Japan), together with NRC (Canada), MOST and ASIAA (Taiwan), and KASI (Republic of Korea), in cooperation with the Republic of Chile. The Joint ALMA Observatory is operated by ESO, AUI/NRAO and NAOJ. A great many organizations and individuals have contributed to the success of the Global Jet Watch observatories and these are listed on http://www.GlobalJetWatch.net but we particularly thank the University of Oxford and the Australian Astronomical Observatory.

\section{ORCID iDs}

Katherine M. Blundell (iD https://orcid.org/0000-00018509-4939

\section{References}

Blundell, K. M., \& Bowler, M. G. 2004, ApJL, 616, L159

Blundell, K. M., \& Bowler, M. G. 2005, ApJL, 622, L129

Blundell, K. M., Schmidtobreick, L., \& Trushkin, S. 2011, MNRAS, 417, 2401

Eikenberry, S. S., Cameron, P. B., Fierce, B. W., et al. 2001, ApJ, 561, 1027

Hjellming, R. M., \& Johnston, K. J. 1980, ApJ, 328, 600

Hjellming, R. M., \& Johnston, K. J. 1981, ApJL, 246, L141

Jeffrey, R. M., Blundell, K. M., Trushkin, S. A., \& Mioduszewski, A. J. 2016, MNRAS, 461, 312

Lockman, F. J., Blundell, K. M., \& Goss, W. M. 2007, MNRAS, 381, 881

Margon, B., 1984, ARA\&A, 22, 507

Migliari, S., Fender, R., \& Méndez, M. 2002, Sci, 297, 1673

Migliari, S., Fender, R. P., Blundell, K. M., Méndez, M., \& van der Klis, M. 2005, MNRAS, 358, 860

Miller-Jones, J. C. A., Migliari, S., Fender, R. P., et al. 2008, ApJ, 682, 1141

Nagai, H., Nakanishi, K., Paladino, R., et al. 2016, ApJ, 824, 132

Rau, U., \& Cornwell, T. J. 2011, A\&A, 532, 71

Roberts, D. H., Wardle, J. F. C., Lipnick, S. L., Selesnick, P. L., \& Slutsky, S. 2008, ApJ, 676, 584

Schnee, S. L., Brogan, C., Espada, D., et al. 2014, Proc. SPIE, 9149, $91490 Z$

Stirling, A. M., Jowett, F. H., Spencer, R. E., et al. 2002, MNRAS, 337, 657

Stirling, A. M., Spencer, R. E., Cawthorne, T. V., \& Paragi, Z. 2004, MNRAS, 354, 1239 